\title{
Exosomes derived from endometriotic stromal cells have enhanced angiogenic effects in vitro
}

\author{
Djana Harp ${ }^{1}$ - Adel Driss ${ }^{1} \cdot$ Sharifeh Mehrabi ${ }^{1}$ - Indrajit Chowdhury ${ }^{1} \cdot$ Wei Xu ${ }^{1}$. \\ Dong Liu $^{2,3}$ • Minerva Garcia-Barrio ${ }^{2,3}$ • Robert N. Taylor ${ }^{4}$ Bert Gold $^{5}$ • \\ Samantha Jefferson ${ }^{6}$ • Neil Sidell ${ }^{7}$ - Winston Thompson ${ }^{1,3}$
}

Received: 30 October 2015 / Accepted: 30 December 2015 /Published online: 3 February 2016

(C) The Author(s) 2016. This article is published with open access at Springerlink.com

\begin{abstract}
Our objective has been to establish a proangiogenic role for exosomes in endometriosis and to determine whether a differential expression profile of cellular and exosomal microRNAs (miRNAs) exists in endometriosis. We performed an in vitro study of human primary endometrial

This work was supported by NIH grants 5U01HD066439 and

1R01HD057235. This publication was also supported by the Research Centers in Minority Institutions Grant Number 8G12MD007602 from the National Institute of Minority Health and Health Disparities (NIMHD) and 8U54MD007588.

The content of this publication is solely the responsibility of the authors and does not necessarily represent the official views of the NIMHD or the $\mathrm{NIH}$.
\end{abstract}

Electronic supplementary material The online version of this article (doi:10.1007/s00441-016-2358-1) contains supplementary material, which is available to authorized users.
Djana Harp
dharp@msm.edu
Adel Driss
adel.driss@gmail.com
Sharifeh Mehrabi
msharifeh@msm.edu
Indrajit Chowdhury
ichowdhury@msm.edu
Wei Xu
wxu@msm.edu
Dong Liu
dliu@msm.edu
Minerva Garcia-Barrio
mgarcia-barrio@msm.edu
Robert N. Taylor
rtaylor@wakehealth.edu

stromal cells (ESCs) and human umbilical vein endothelial cells (HUVECs). We isolated and characterized exosomes from ESCs from five endometriosis patients and five phasematched controls. Exosomes were characterized by transmission electron microscopy and NanoSight technology. MiRNA
Bert Gold

bgold@natera.com

Samantha Jefferson

sjefferson13@student.gsu.edu

Neil Sidell

nsidell@emory.edu

Winston Thompson

wthompson@msm.edu

1 Department of Obstetrics and Gynecology, Morehouse School of Medicine, 720 Westview Drive, SW, Atlanta GA 30310, USA

2 Cardiovascular Research Institute, Morehouse School of Medicine, 720 Westview Drive, SW, Atlanta GA 30310, USA

3 Department of Physiology, Morehouse School of Medicine, 720 Westview Drive, SW, Atlanta GA 30310, USA 
was assessed by deep sequencing and reverse transcription with quantitative polymerase chain reaction. Exosome uptake studies were achieved by means of confocal microscopy. The pro-angiogenic experiments were executed by treating HUVECs with ESC-derived exosomes. We observed differential profiles of exosomal miRNA expression between exosomes derived from endometriosis lesion cells and diseased eutopic stromal cells compared with exosomes derived from control ESCs. We also demonstrated autocrine cellular uptake of exosomes and paracrine functional angiogenic effects of exosomes on HUVECs. The results of this study support the hypothesis that exosomes derived from ESCs play autocrine/paracrine roles in the development of endometriosis, potentially modulating angiogenesis. The broader clinical implications are that Sampson's theory of retrograde menstruation possibly encompasses the finding that exosomes work as intercellular communication modulators in endometriosis.

Keywords Exosomes · Endometrial stromal cells ·

Angiogenesis $\cdot$ MicroRNA $\cdot$ Infertility

\section{Introduction}

Endometriosis is a benign gynecological disorder characterized by the presence of endometrial glandular tissue and stroma outside of their normal uterine location. Up to $10 \%$ of reproductive-age women in the United States are estimated to have endometriosis, which is associated with debilitating pain, menstrual irregularities, and infertility in as many $30 \%$ of the affected women (Bulun 2009; Verkauf 1987). The precise etiology of endometriosis remains to be elucidated. Commonly accepted theories of the causes of endometriosis include retrograde menstruation, coelomic metaplasia, and lymphatic and vascular dissemination (Macer and Taylor 2012). Patients with endometriosis often have delayed (611 years) diagnosis because symptoms are variable and often mimic other gynecological diseases (Fassbender et al. 2015; Leone Roberti Maggiore et al. 2016; Nogales et al. 1993). A definitive diagnosis of endometriosis requires surgery (Gordts et al. 2015). The development of new ways of diagnosis by using exosomal biomarkers might enable earlier diagnosis of the disease (Fassbender et al. 2015).

$4 \quad$ Department of Obstetrics and Gynecology, Wake Forest School of Medicine, 1 Medical Center Boulevard, Winston-Salem NC 27157, USA

5 Center for Cancer Research, National Cancer Institute, Frederick MD 21702, USA

6 Georgia State University, P.O. Box 3965, Atlanta GA 30302, USA

7 Department of Gynecology \& Obstetrics, Emory University School of Medicine, 1639 Pierce Dr., WMB 4303, Atlanta GA 30322, USA
Angiogenesis, the formation of new blood vessels, has increasingly been recognized as a key pathological factor in endometriosis. Human endometrial tissue is known to be angiogenic throughout the menstrual cycle (Maas et al. 2001). Sampson's theory of retrograde menstruation posits that endometrial tissue travels retrogradely through the Fallopian tubes and is deposited into the peritoneal cavity, where it establishes a blood supply, proliferates, and forms an implant that is clinically recognized as a lesion (Sampson 1927). Women with endometriosis have been found to have increased retrograde menstruation (Halme et al. 1984). Successful implantation of endometriotic lesions depends on neovascularization, fibrosis, adhesion formation, apoptosis evasion, and neuronal infiltration (Rocha et al. 2013). The exact mechanisms underlying the sprouting and sustainment of endometriosis neovascularization need further clarification. Recent studies, however, demonstrate that inhibitors of angiogenesis block the progression of endometriosis (Edwards et al. 2013; Nap et al. 2004, Wang et al. 2014).

Exosomes have been found to play physiological roles as mediators of intercellular cell signaling between neighboring cells and even amongst distant tissues, and they may act independently but synergistically with soluble growth factors and hormones (Colombo et al. 2014). Exosomes are vesicles ranging from 30 to $150 \mathrm{~nm}$ in diameter, are derived from the fusion of multivesicular bodies with the plasma membrane, and are secreted by a variety of living cells; they are composed of a lipid bilayer membrane and contain functionally active proteins, $m R N A$ and microRNA (miRNA) that can be released, rendering them important mediators of intercellular communication (Colombo et al. 2014). The recent discovery of these extracellular organelles containing genetic cargo with a unique signature raises the hope that exosomes will serve as diagnostic markers or therapeutic vehicles (Krause et al. 2015; Momen-Heravi et al. 2015; Tang and Wong 2015).

Among all molecules, the miRNAs that are contained within exosomes (Valadi et al. 2007) could affect gene expression and function in target cells, including vascular endothelial cells. Several miRNAs, such as $m i R-21$ and $m i R-126$, have been shown previously to play critical roles in angiogenesis (Wang and Olson 2009). We have chosen to screen these two miRNAs because, in previous literature, they have been widely used by the scientific community as pro-angiogenic indicators in other systems (Bao et al. 2012; Guduric-Fuchs et al. 2012; Xu et al. 2015).

Additionally, endometrial exosomes released into the uterine cavity can transfer information either to the blastocyst or to other cells in the endometrium to influence implantation $(\mathrm{Ng}$ et al. 2013). Our hypothesis is that exosomes released from endometriotic stromal cells contribute to the pathogenesis of endometriosis by packaging and delivering specific miRNAs in an autocrine and paracrine fashion. In this study, we have examined exosomes as delivery vehicles for pro-angiogenic miRNA in endometriosis. 


\section{Materials and methods}

\section{Human subjects and tissue acquisition}

The study was approved by the institutional review boards of the Emory University School of Medicine and Morehouse School of Medicine. The primary endometrial stromal cells (ESCs) were obtained from normally cycling, reproductiveage women undergoing surgery for benign gynecological conditions with normal endometrium (NE, $n=5)$ and 5 women with endometriosis who provided eutopic endometrial biopsies (EE, $n=5)$ plus specimens from their endometriosis implants (EI, $n=5$ ). For NE controls, endometrial tissues were obtained from patients undergoing surgery for clinical indications, typically infertility, pelvic pain, or suspicion of a pelvic mass, or some combination of findings under protocols approved by the Institutional Review Boards of the Emory University School of Medicine and Morehouse School of Medicine. All the subjects selected were women who had regular menstrual cycles and who had not received hormonal therapy for at least 3 months before surgery (Yu et al. 2014). Absence of endometriosis in the NE group was confirmed after surgical examination of the abdominal and pelvic cavity. Specimens from controls were obtained from normally cycling women undergoing surgery for benign gynecological conditions in which no endometriosis or evidence of endometrial abnormalities was visible. Among the five control subjects, subserosal fibroids were noted in four women, and none were greater than $3 \mathrm{~cm}$ in diameter. Endometriosis patients were identified at surgery by expert laparoscopists familiar with the varied appearance of the lesions. Although the subjects were not age-matched, their mean ages were not significantly different (Control, 40.4 \pm 5.9 ; Endometriosis, 34.0 $\pm 7.8 ; P=0.53)$. The secretory menstrual phase according to the day of the reproductive cycle was selected for all biopsies to maximize consistency and was confirmed by histological examination of the endometrial tissues. Written informed consent was obtained prior to surgical removal of endometriotic lesion tissue and endometrial biopsies.

\section{Cell cultures and reagents}

Primary ESCs were prepared from human tissue biopsies according to our published procedure (Ryan et al. 1994). All cultures (passages 3-5) were grown in complete medium to 70-90 \% confluence in DMEM/Ham's F-12 supplemented with $10 \%$ fetal bovine serum (FBS), $1 \%$ non-essential amino acids, $1 \%$ sodium pyruvate, and $1 \%$ penicillin streptomycin and were incubated at $37{ }^{\circ} \mathrm{C}$ and $5 \% \mathrm{CO}_{2}$. Human umbilical vein endothelial cells (HUVECs) were purchased from Lonza (Walkersville, Md., USA) and were cultured in EBM-2 media supplemented with EGM-2 MV cocktail (Lonza) together with $1 \%$ penicillin and streptomycin.

\section{Isolation of exosomes}

Once the desired confluence was obtained, the culture media were removed, and ESCs were washed twice with $5 \mathrm{ml}$ sterile phosphate-buffered saline (PBS). The ESCs were then cultured for an additional $48 \mathrm{~h}$ with growth media containing exosome-depleted FBS. Exosome-depleted FBS was obtained by ultracentrifugation of FBS at $100,000 \mathrm{~g}$ for $16 \mathrm{~h}$ at $4{ }^{\circ} \mathrm{C}$. The exosomal fraction from $5 \mathrm{ml}$ culture media was isolated by the Total Exosome Isolation kit (Invitrogen) according to the manufacturer's recommendations. First, the collected cell culture media were centrifuged at $2000 \mathrm{~g}$ for $30 \mathrm{~min}$ at room temperature to remove cells and debris. Second, a half-volume of the exosome isolation solution was added to cell-free culture media, and samples were refrigerated at $4{ }^{\circ} \mathrm{C}$ overnight. The mixture was centrifuged at $10,000 \mathrm{~g}$ for $1 \mathrm{~h}$ at $4{ }^{\circ} \mathrm{C}$, and the supernatant was removed by aspiration. The pellet was resuspended in $1 \times \mathrm{PBS}$ and stored at $-80^{\circ} \mathrm{C}$ or directly processed for miRNA extraction ( $\mathrm{Li}$ et al. 2015).

\section{Identification of nanoparticles by nanoparticle tracking analysis}

Nanoparticle tracking analysis (NTA) measurements were performed by using a NanoSight NS500 instrument (NanoSight NTA 2.3 Nanoparticle Tracking and Analysis Release Version Build 0025). The size distribution and quantification of exosome preparations were analyzed by measuring the rate of Brownian motion with a NanoSight LM10 system (NanoSight, Wiltshire, United Kingdom) equipped with fast video capture and particle-tracking software. Purified exosomes from NE, EE, and EI samples were diluted in $500 \mu \mathrm{l}$ of a solution of $1 \times \mathrm{PBS} / 5 \mathrm{mM}$ EDTA and disaggregated by using a syringe and needle (29-gauge). After this procedure, the sample was injected into a NanoSight sample cubicle. The mean \pm SD size distribution of ESC exosomes was determined, and the mean number of particles per milliliter was compared between endometriosis patients (exosomes derived from EE and EI ESCs) and exosomes derived from eutopic NE cells of healthy subjects (Riches et al. 2014).

\section{Exosome visualization by transmission electron microscopy}

Exosome suspension was loaded into a carbon-coated electron microscopy grid. The sample was fixed with $2.5 \%$ glutaraldehyde in $0.1 \mathrm{M}$ cacodylate buffer for $2 \mathrm{~h}$ at $4{ }^{\circ} \mathrm{C}$, followed by a second fixation with $1 \%$ osmium tetroxide in $0.1 \mathrm{M}$ cacodylate buffer for $1 \mathrm{~h}$ at $4{ }^{\circ} \mathrm{C}$. After three washes in distilled $\mathrm{H}_{2} 0$, the sample was stained with $0.5 \%$ aqueous uranyl acetate for $2 \mathrm{~h}$ at room temperature. Transmission electron microscopy samples were observed by using a JEOL $1200 \mathrm{EX}$ instrument. 


\section{Exosome labeling}

Exosomes were first obtained from non-labeled ESCs as described above. The protein content of the exosomes was adjusted to $1.4 \mu \mathrm{g} / \mathrm{ml}$ prior to labeling. Exosomal membrane was labeled with BODIPY TR ceramide, according to the manufacturer's protocol (Molecular Probes/Invitrogen Life Technologies). Briefly, exosome pellets were re-suspended in $100 \mu \mathrm{l}$ PBS and stained with $10 \mu \mathrm{mol} / \mathrm{L}$ BODIPY TR ceramide with 594Alexa-Fluor (red) fluorescence. Excess fluorescent dye was removed by using Exosome Spin Columns (Life Technologies).

\section{Confocal microscopy and imaging}

Cells were cultivated on chamber slides, treated with a final concentration of $28 \mathrm{ng} / \mathrm{ml}$ labeled exosomes (red) for $20 \mathrm{~min}$, fixed with $4 \%$ paraformaldehyde at room temperature for $20 \mathrm{~min}$, permeabilized with ice-cold acetone at room temperature for $5 \mathrm{~min}$, and then stained with fluorescent 488-Phalloidin (green) for actin using the manufacturer's recommendations (Molecular Probes/ Invitrogen Life Technologies). Fluorescence images were collected on an inverted microscope (Zeiss LSM700) via a $40 \times$ objective. Zeiss acquisition parameters, including exposure, focus, illumination, and $\mathrm{Z}$ stack projection, were controlled by ZEN 2 software (Carl Zeiss Microscopy). For the analysis of the cellular internalization of exosomes, images were also deconvolved by using ZEN 2 software (Chu et al. 2012).

\section{Endothelial tube formation assay}

Sub-confluent HUVECs were harvested, resuspended in medium, and treated with the indicated concentration of exosomes or with PBS as a negative control. The exosome protein concentration was measured by the Coomassie Plus (CP) Protein Assay (Thermo Scientific). Treatment of cells consisted of $50 \mu \mathrm{g}$ total excreted exosomes extracted from eutopic endometriosis and control endometrial cells. This suspension was seeded $(70,000$ cells/well) in growthfactor-reduced Geltrex Basement Membrane Matrix (Gibco) on a 96-well plate (BD Bioscience) and incubated up to $24 \mathrm{~h}$ at $37{ }^{\circ} \mathrm{C}$ with $5 \% \mathrm{CO}_{2}$. Tube formation was examined under an inverted microscope and photographed at $40 \times$ magnification. Cumulative tube length was measured by using ImageJ software (Schneider et al. 2012). Results are shown as the mean and standard errors of triplicate experiments (Swift et al. 2010).
Micro-RNA extraction, deep sequencing, and reverse transcription with quantitative polymerase chain reaction

Micro-RNA was extracted from exosomes and cells by using the mirVana miRNA isolation kit (Life Technologies) according to the manufacturer's protocol. $R N A$ concentration and relative quality were determined by using a Nanodrop Spectrophotometer (Thermo Scientific). Samples were deemed acceptable if 260/280 ratios were greater than $1.6 ; 260 / 230$ ratios were greater than 1.0. miRNA $(1 \mu \mathrm{g})$ was reverse-transcribed by using the TaqMan MicroRNA Reverse Transcription Kit (Applied Biosystems) following the manufacturer's protocol. Primers for hsa-miR-21-5p (Cat. no. 4427975000397), hsa-miR-126-5p (Cat. no. 4427975-000451), and U6 snRNA (Cat. no. 4427975-001973) were used from miRNA kits (Applied Biosystems), and real-time quantitative polymerase chain reaction (qPCR) was performed by using the LC480 Lightcycler thermocycler (Roche). snRNA U6 was used as an endogenous control to normalize expression data. All samples were run in triplicate, and the average value was used to calculate the fold change values. The delta-delta of average $\mathrm{Ct} / \mathrm{Cp}$ value method was used to calculate the fold change values among samples as previously described (Scarlett et al. 2014). Deep sequencing of total miRNAs was carried out by SeqMatic LLC (Fremont, Calif., USA). Illumina sequencing libraries were generated by using the SeqMatic TailorMix miRNA sample preparation kit. Sequencing was performed by using the Illumina Genome Analyzer IIx with a $1 \times 36$ bp single-end read. Data was demultiplexed by index barcode, and fastq files were generated by using Illumina pipeline (CASAVA). For data quality control, sequence reads were trimmed of the Illumina adapters by using fastx clipper. Reads shorter than $15 \mathrm{bp}$ were removed from subsequent analyses. For data analysis, clean sequence reads were mapped to the human genome (hg19) and to miRBase version 20 by using Bowtie. Parameters were modified to allow for up to one mismatch. Reads mapping to known miRNAs were counted. Differential expression and $P$-value estimation were performed by using DESeq for $\mathrm{R}$ statistical programming language (Ge et al. 2014).

\section{Statistics}

Real-time qPCR results were expressed as means \pm standard deviation (SD) from at least three separate experiments performed in triplicate, unless otherwise stated. Differences between means among the treatment groups were analyzed by using the Student two-tailed $t$-test or one-way analysis of variance (ANOVA) with Holm-Sidak post-tests method where appropriate. Quantitative analysis of branching length in the 
angiogenesis assay was performed with Pearson correlation analysis. A $P$-value of $<0.05$ was considered significant. SigmaPlot (version 11.0) and MS Excel software for Windows were used for statistical analysis.

\section{Results}

\section{Identification of ESC-derived exosomes in culture media}

The ESC exosome purification procedure was validated by using electron microscopy and NanoSight analysis. Figure 1a shows an example of a transmission electron micrograph of a representative exosome preparation. Further characterization of exosomes, including size measurement and quantification, from healthy (Fig. 1b-d, Supplementary video 1) and endometriosis subjects was performed by using NanoSight analysis, which demonstrated the purity of the vesicles, with a peak size at $35 \mathrm{~nm}$. This confirmed that the ESCs of control and endometriosis subjects released exosomal vesicles into their conditioned medium. No measurable differences were observed in size or yield (Fig. 1e, f).

\section{Autocrine internalization of exosomes by ESCs}

We hypothesized that exosomes work in an autocrine/ paracrine fashion and can be internalized by nearby cells to affect function in the uterine microenvironment or peritoneal cavity. The internalization of labeled purified exosomes was visualized by confocal fluorescence microscopy in ESCs (Fig. 2a-g, Supplementary Fig. S1, Supplementary video 2). We observed that the internalization of exosomes occurred within $2 \mathrm{~min}$ of exposure to treatment, suggesting a fast uptake mechanism (Fig. 2h, i). In these experiments (Fig. 2), we observed the uptake of exogenous purified NE exosomes by NE ESCs. Similar results were found when we used EE exosomes on NE cells, and when we used EE cells. The findings support the ability of exosomes to influence cell behavior via autocrine and/or paracrine routes.
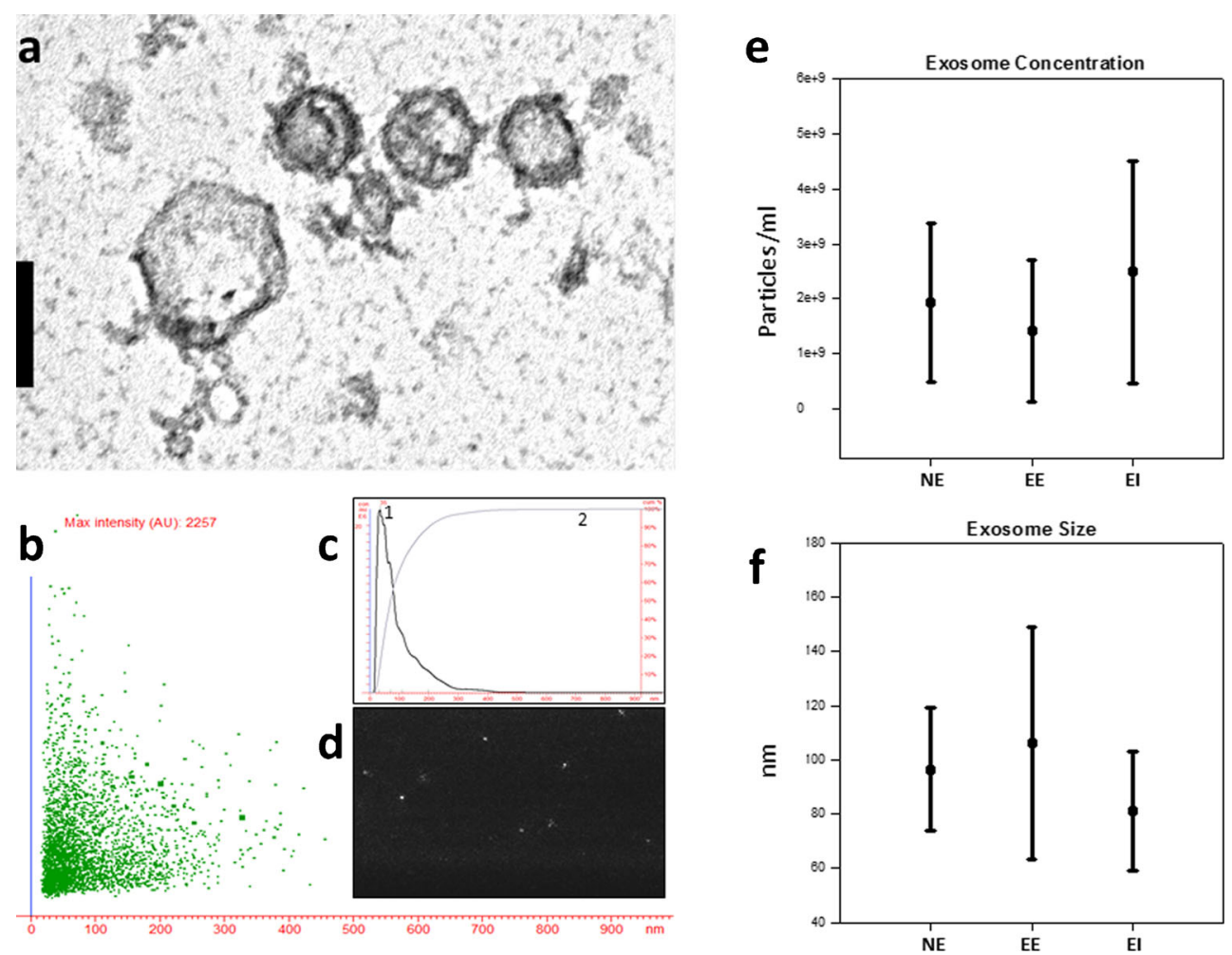

Fig. 1 a Transmission electron microscopy of exosomes from control endometrial stromal cells (ESCs). Bar $100 \mathrm{~nm}$. b Scatter plot graph of exosomes by using Nanosight technology shows that the majority of exosomes are between 20 and $200 \mathrm{~nm}$ in size with a peak at $35 \mathrm{~nm}$ ( $X=$ particle size, $Z=$ count $/ \mathrm{ml}$ ). c Curve 1 describes the relationship between particle number distribution (concentration/ml; left $Y$ axis) and particle size (in nm; $X$ axis); curve 2 describes the correlation between the cumulative percentage distribution of particles (percentile; right $Y$ axis) and particle size ( $X$ axis). d Still figure of exosome scatter nanoparticle tracking in a NanoSight video (Supplementary video 1). e Repeated- measure analysis of variance showing scatter plot column comparison of means of exosome concentrations between the three groups ( $N E$ normal endometrium, $E E$ eutopic endometrium, $E I$ endometriosis implants). No measurable differences between the three groups were observed $(P=0.655)$. f Scatter plot column comparison of means of exosome sizes between the three groups. No measurable differences between the three groups were observed $(P=0.529)$. Error bars are the standard deviations of the different readings from different isolations from T-75 flasks after $24 \mathrm{~h}$ of changing to exosome-free medium 

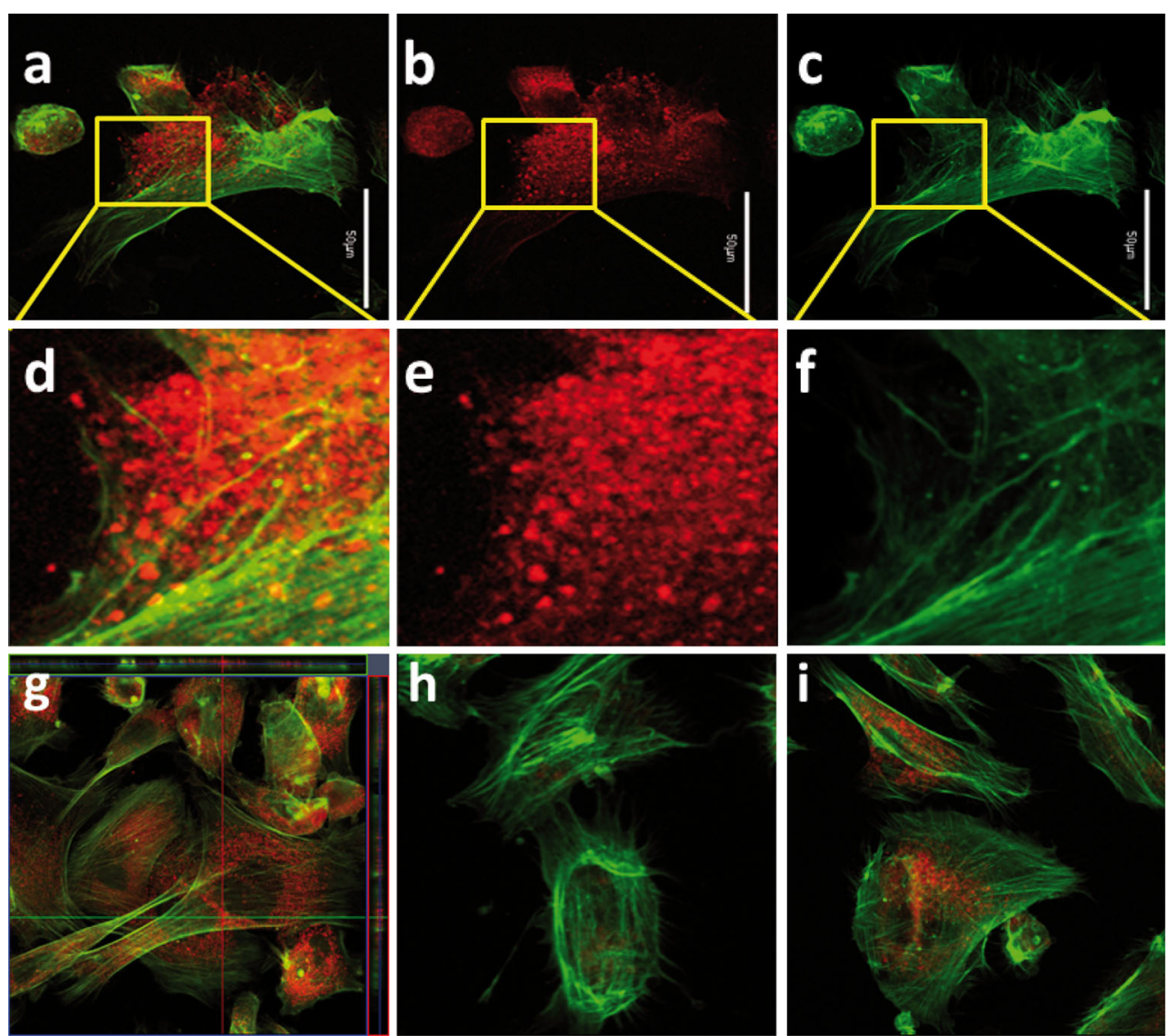

Fig. 2 Uptake of exosomes by NE ESCs. The protein equivalent of $28 \mathrm{ng} / \mathrm{ml}$ labeled exosomes (red) was incubated for 20 min with ESCs, which were then fixed in $4 \%$ paraformaldehyde at room temperature for $20 \mathrm{~min}$, subsequently permeabilized with ice-cold acetone at room temperature for $5 \mathrm{~min}$, and finally labeled with 488-Phalloidin (green). Similar results were obtained when we used NE and EE exosomes, and when we used EE cells. a ESCs labeled with 488-Phalloidin (green). b Exosomes labeled with 594-Alexa-Fluor (red). c Merged image. a-c

\section{Pro-angiogenic effects of exosomes derived from ESCs}

HUVECs plated on Geltrex Basement Membrane Matrix in medium treated with exosomes extracted from eutopic endometriosis ESC medium had greater tube formation compared with those treated with exosomes extracted from control ESC medium (Fig. 3a, b). A significant increase $(P=0.033)$ of total segment branch length was noted on HUVECs treated with exosomes extracted from endometriosis cells compared with control cells (Fig. 3c).

\section{Differential expression of exosomal pro-angiogenic miRNAs}

Deep sequencing data also showed that several miRNAs extracted from exosomes of NE and EE were differentially expressed between the two groups (Fig. 3d). The deep sequencing results led to the identification of several miRNAs
Magnification: $40 \times$. d-f Higher magnification of boxed areas in a-c. g Z-stack slices running apical to basolateral in ESCs showing the ZX (upper box) and ZY (right side box) cross sections of the selected cells (Supplementary video 2) showing that the exosomes (red) are located inside the ESCs and not on the top or bottom. $\mathbf{h}$ Uptake by the ESCs of exosomes after 2 min of treatment. i Uptake by the ESCs of exosomes after 8 min of treatment

that have yet to be characterized and confirmed in a larger patient sample size. As angiogenesis has been recognized to be a key pathological factor in endometriosis, we found, from our deep sequencing heat-map data, that some miRNAs could be predicted to be involved in the angiogenesis process. Since our endothelial tube assay showed proangiogenic effects in EE-treated HUVECs, we analyzed two known pro-angiogenic miRNAs (miR-21 and miR$126)$ to determine whether differential expression occurred. We investigated the expression in total miRNA extracted from exosomes secreted by NE, EE, and EI ESCs. Whereas the data did not reveal significant differences in miR-126 among samples, EI cells showed an 11-fold increase in $m i R-21$ expression versus the healthy control eutopic ESC exosomes $(P<0.0001$; Fig. $3 \mathrm{e}, \mathrm{f})$. Intracellular expression levels of $m i R-21$ and $m i R-126$ extracted from the producing cells in culture showed no significant differences between groups (Fig. 3g, h). 

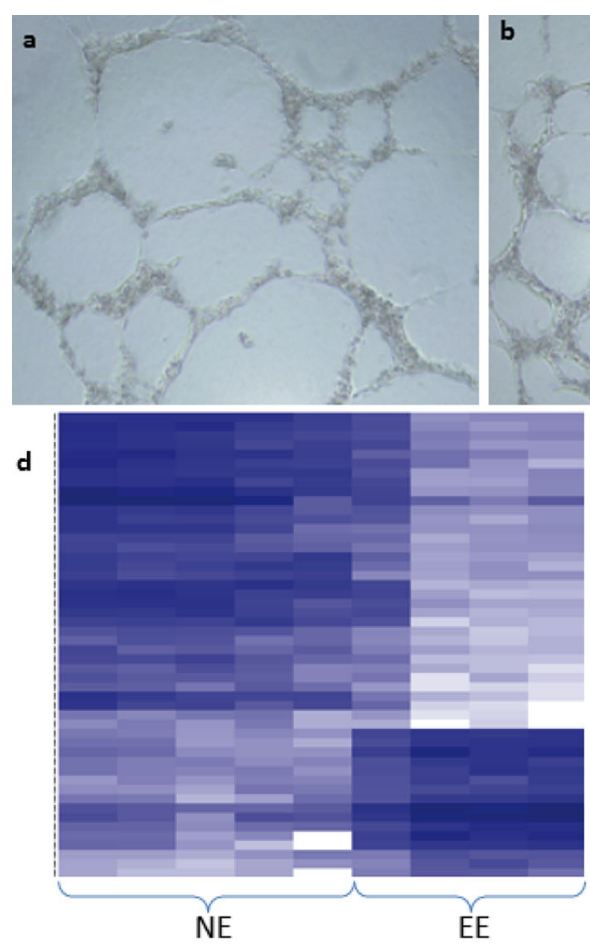

Fig. 3 a HUVECs treated with exosomes extracted from control cells at $24 \mathrm{~h}$. b HUVECs treated with exosomes extracted from endometriosis patient cells at $24 \mathrm{~h}$. c Significant increase of total segment branch length is noted on HUVECs treated with exosomes extracted from EE cells compared to those from NE cells (error bar SD). d Heat-map from deep-sequencing depicting the most significant differential expression of miRNAs extracted from exosomes of NE and from EE. e, f $m i R-21$ and $m i R-126$ expression, respectively, revealed by reverse transcription and quantitative polymerase chain reaction (RT-qPCR) of RNA extracted

\section{Discussion}

Angiogenesis is necessary for the establishment and proliferation of endometriotic lesions (Shifren et al. 1996). Many molecules and various mechanisms involved in lesion angiogenesis are currently under study (Araldi et al. 2015; Kazanis et al. 2015; Li et al. 2015).

We have demonstrated exosome release from ESCs and confirmed the presence of exosomes by morphological analysis and dimensions. We have shown that the exosomes are rapidly uptaken by ESCs in vitro (Fig. 2). No morphological difference has been noted following the uptake of exosomes from NE cells and from EE cells by either cell type. We postulate that specific surface ligands allow the highly efficient and targeted uptake of these vesicles by recipient cells, as has been shown in other cell types (Schneider and Simons 2013). We hypothesize that ESC-released exosomes are taken up by and have a pro-angiogenic effect on endothelial cells that helps to promote tube formation. We have further shown differential exosomal angiogenic activity on HUVECs in the in
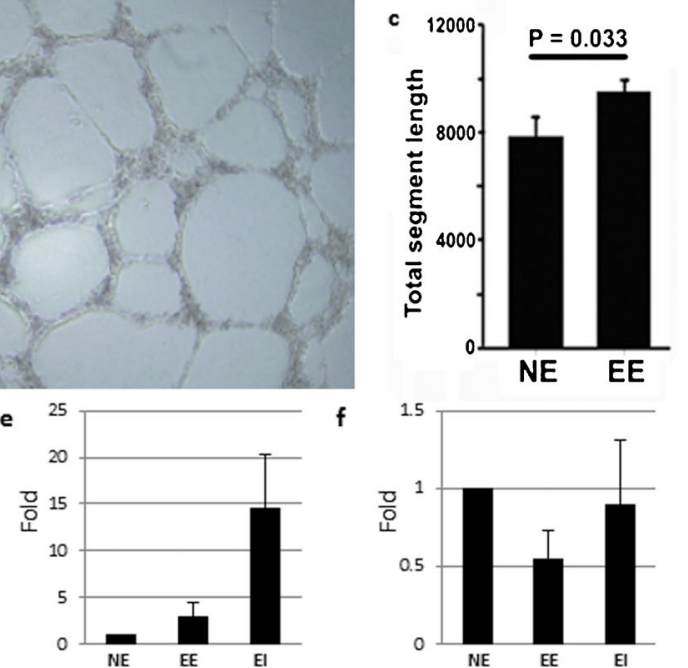

miR-21 Expression in Exosomes

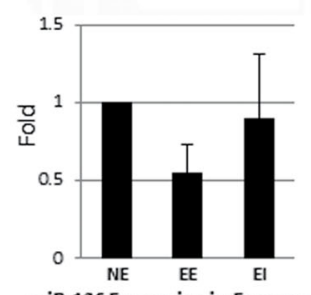

miR-126 Expression in Exosomes
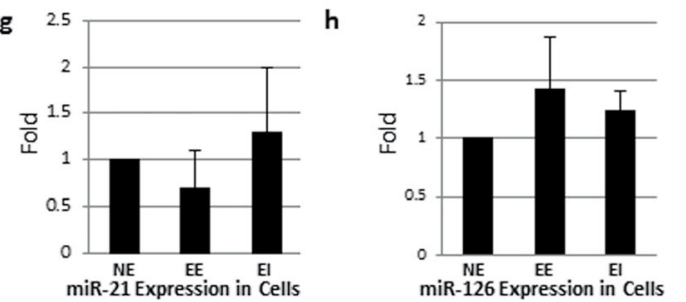

from exosomes from NE, EE, and EI samples. EI cells showed an 11-fold increase in $m i R-21$ expression versus the healthy control eutopic ESC exosomes $(P<0.0001)$. No significant difference in miR-126 expression was found among samples. $\mathbf{g}, \mathbf{h} m i R-21$ and $m i R-126$ expression, respectively, revealed by RT-qPCR of RNA extracted from cells of NE, EE, and EI samples. No significant differences between groups were shown between intracellular expression levels of miR-21 and miR-126 extracted from the producing cells in culture

vitro Matrigel assay. Endothelial cells are integrally necessary for the formation of new blood vessels. We have observed increased branching when the HUVECs are treated with EE exosomes compared with NE exosomes (Fig. 3a-c). These data suggest that exosomes play a role in promoting endothelial cell formation and angiogenesis in endometriosis. Exosomes from a variety of other cell types have been shown to exert a pro-angiogenic effect on endothelial cells and on stromal cells (Mineo et al. 2012; Paggetti et al. 2015; Pascucci et al. 2014). From our data, we propose that exosomes can play an important mediator role in the angiogenesis process in vitro in a paracrine fashion.

In the present study, we show that ESCs release biologically functional exosomes that can facilitate angiogenesis in a validated in vitro assay. Exosomes carry a variety of bioactive molecules: RNAs, DNAs, proteins, and lipids (Colombo et al. 2014). The various types of response in target cells induced by exosomes derived from parent cells are probably determined by their miRNA content (Pascucci et al. 2014; Shabbir et al. 2015). Our deep sequencing data suggest that endometriosis 
patients have a differential expression of exosomal miRNA patterns compared with controls (Fig. 3d). This signifies that differential pathogenic roles and altered mechanisms are involved in exosomal intercellular communication in the peritoneal and intrauterine milieu.

Our results indicate that at least one of these factors, namely the pro-angiogenic $m i R N A, m i R-21$, is differentially expressed in lesion exosomes of endometriosis-derived samples (EI) relative to normal (NE) exosomes. Transferred exosomal miRNA can reprogram the recipient cells. The linkage between exosomal miRNAs and their effects in the recipient cells remains to be fully elucidated. However, evidence to date indicates that exosomes are powerful mediators of intercellular communication (Tang and Wong 2015). We propose that the exposure of endothelial cells to ESC exosomes leads to angiogenesis, with some of the effects possibly being attributable to the overexpression of $m i R-21$. Exosomes containing $m i R-21$ have been previously reported to stimulate cell angiogenesis in other systems (Bao et al. 2012; GuduricFuchs et al. 2012; Xu et al. 2015). We speculate that exosome-derived miRNAs are potential angiogenic targets that are relevant to endometriosis sequelae such as abnormal implantation and infertility. Thus, these exosome-specific miRNAs would be present in the uterine and peritoneal microenvironments mediating cross-talk essential for the constellation of secondary effects of endometriosis. The work
Fig. 4 Suggested model depicting exosome shedding in the endometrial cavity working in an autocrine, paracrine, and endocrine manner. Exosomes may also be shed in a retrograde fashion with menstrual flow and be taken up by cells within the peritoneal cavity or shed by stromal cells in the menstrual flow

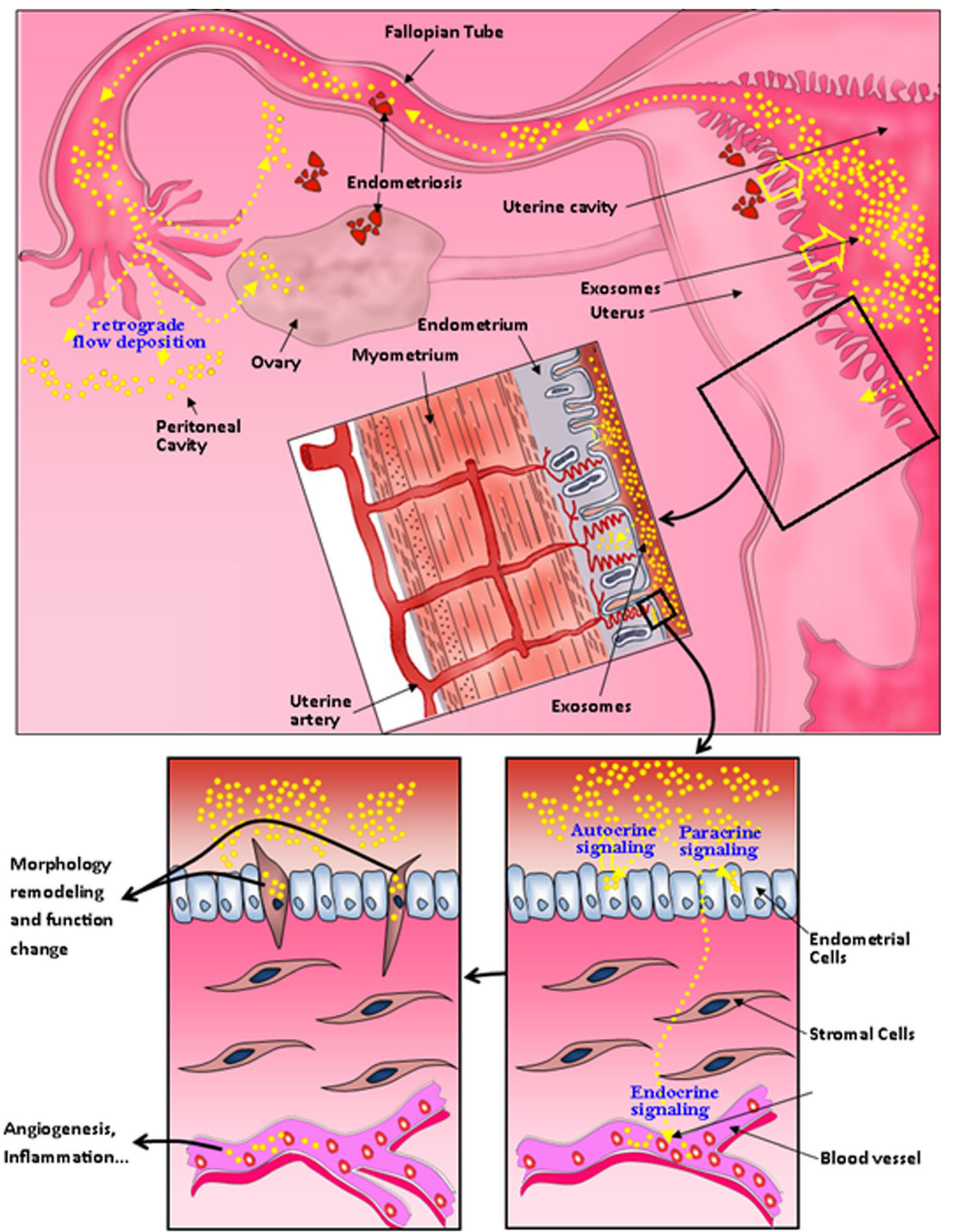


presented here sets the basis for a path toward the discovery of novel effective biomarkers for early diagnostics and potential new drug targets.

This study supports the hypothesis that exosomes derived from endometriotic stromal cells play autocrine/paracrine roles in the endometrial and peritoneal microenvironments, modulating angiogenesis. Figure 4 shows our suggested model of action of exosomes in the uterine microenvironment. Additionally, exosomes can migrate further into other tissues through blood vessels (endocrine; De Toro et al. 2015; Ng et al. 2013; Salomon et al. 2014; Simpson et al. 2008) and be transported to the peritoneal cavity through a retrograde flow deposition system. Furthermore, these lesion exosomes might modify angiogenesis in the local peritoneal environment.

The broader clinical implications discussed are built on Sampson's theory of retrograde menstruation, a theory that supports the idea that exosomes work as intercellular communication modulators in endometriosis. We suggest a model in which exosomes secreted from diseased endometrial cells stimulate the pro-angiogenic propensity of nascent lesions in the peritoneum to adopt a new vasculature and cause inflammatory changes that lead to pelvic pain and infertility. This pathogenic intercellular transfer of genetic material mediated by exosome carriers could pave the road to new diagnostic and therapeutic tools in the field of reproductive biology.

Acknowledgments The authors thank Mr. Lawrence Brako and Dr. Woo Kuen Lo for assistance with the electron microscopy, and Dr. Gregory Adams for technical assistance with the confocal microscopy at the MSM Core Facility.

\section{Compliance with ethical standards}

Conflict of interest The authors declare that they have no conflict of interest.

Open Access This article is distributed under the terms of the Creative Commons Attribution 4.0 International License (http:// creativecommons.org/licenses/by/4.0/), which permits unrestricted use, distribution, and reproduction in any medium, provided you give appropriate credit to the original author(s) and the source, provide a link to the Creative Commons license, and indicate if changes were made.

\section{References}

Araldi E, Chamorro-Jorganes A, Solingen C van, Fernandez-Hernando C, Suarez Y (2015) Therapeutic potential of modulating microRNAs in atherosclerotic vascular disease. Curr Vasc Pharmacol 13:291-304

Bao F, Wu P, Xiao N, Qiu F, Zeng QP (2012) Nitric oxide-driven hypoxia initiates synovial angiogenesis, hyperplasia and inflammatory lesions in mice. PLoS One 7:e34494

Bulun SE (2009) Endometriosis. N Engl J Med 360:268-279
Chu L, Zhu T, Liu X, Yu R, Bacanamwo M, Dou Z, Chu Y, Zou H, Gibbons GH, Wang D, Ding X, Yao X (2012) SUV39H1 orchestrates temporal dynamics of centromeric methylation essential for faithful chromosome segregation in mitosis. J Mol Cell Biol 4:331340

Colombo M, Raposo G, Thery C (2014) Biogenesis, secretion, and intercellular interactions of exosomes and other extracellular vesicles. Annu Rev Cell Dev Biol 30:255-289

De Toro J, Herschlik L, Waldner C, Mongini C (2015) Emerging roles of exosomes in normal and pathological conditions: new insights for diagnosis and therapeutic applications. Front Immunol 6:203

Edwards AK, Nakamura DS, Virani S, Wessels JM, Tayade C (2013) Animal models for anti-angiogenic therapy in endometriosis. $\mathrm{J}$ Reprod Immunol 97:85-94

Fassbender A, Burney RO, O DF, D'Hooghe T, Giudice L (2015) Update on biomarkers for the detection of endometriosis. Biomed Res Int 2015:130854

Ge Q, Zhou Y, Lu J, Bai Y, Xie X, Lu Z (2014) miRNA in plasma exosome is stable under different storage conditions. Molecules 19:1568-1575

Gordts S, Puttemans P, Gordts S, Brosens I (2015) Ovarian endometrioma in the adolescent: a plea for early-stage diagnosis and full surgical treatment. Gynecol Surg 12:21-30

Guduric-Fuchs J, O'Connor A, Cullen A, Harwood L, Medina RJ, O'Neill CL, Stitt AW, Curtis TM, Simpson DA (2012) Deep sequencing reveals predominant expression of miR-21 amongst the small non-coding RNAs in retinal microvascular endothelial cells. J Cell Biochem 113:2098-2111

Halme J, Hammond MG, Hulka JF, Raj SG, Talbert LM (1984) Retrograde menstruation in healthy women and in patients with endometriosis. Obstet Gynecol 64:151-154

Kazanis I, Feichtner M, Lange S, Rotheneichner P, Hainzl S, Oller M, Schallmoser K, Rohde E, Reitsamer HA, Couillard-Despres S, Bauer HC, Franklin RJ, Aigner L, Rivera FJ (2015) Lesioninduced accumulation of platelets promotes survival of adult neural stem/progenitor cells. Exp Neurol 269:75-89

Krause M, Samoylenko A, Vainio SJ (2015) Exosomes as renal inductive signals in health and disease, and their application as diagnostic markers and therapeutic agents. Front Cell Dev Biol 3:65

Leone Roberti Maggiore U, Ferrero S, Mangili G, Bergamini A, Inversetti A, Giorgione V, Vigano P, Candiani M (2016) A systematic review on endometriosis during pregnancy: diagnosis, misdiagnosis, complications and outcomes. Hum Reprod Update 22:70-103

Li M, Rai AJ, Joel DeCastro G, Zeringer E, Barta T, Magdaleno S, Setterquist R, Vlassov AV (2015) An optimized procedure for exosome isolation and analysis using serum samples: application to cancer biomarker discovery. Methods 87:26-30

Maas JW, Groothuis PG, Dunselman GA, Goeij AF de, Struyker Boudier HA, Evers JL (2001) Endometrial angiogenesis throughout the human menstrual cycle. Hum Reprod 16:1557-1561

Macer ML, Taylor HS (2012) Endometriosis and infertility: a review of the pathogenesis and treatment of endometriosis-associated infertility. Obstet Gynecol Clin N Am 39:535-549

Mineo M, Garfield SH, Taverna S, Flugy A, De Leo G, Alessandro R, Kohn EC (2012) Exosomes released by K562 chronic myeloid leukemia cells promote angiogenesis in a Src-dependent fashion. Angiogenesis 15:33-45

Momen-Heravi F, Saha B, Kodys K, Catalano D, Satishchandran A, Szabo G (2015) Increased number of circulating exosomes and their microRNA cargos are potential novel biomarkers in alcoholic hepatitis. J Transl Med 13:261

Nap AW, Griffioen AW, Dunselman GA, Bouma-Ter Steege JC, Thijssen VL, Evers JL, Groothuis PG (2004) Antiangiogenesis therapy for endometriosis. J Clin Endocrinol Metab 89:1089-1095

Ng YH, Rome S, Jalabert A, Forterre A, Singh H, Hincks CL, Salamonsen LA (2013) Endometrial exosomes/microvesicles in 
the uterine microenvironment: a new paradigm for embryoendometrial cross talk at implantation. PLoS One 8:e58502

Nogales FF, Martin F, Linares J, Naranjo R, Concha A (1993) Myxoid change in decidualized scar endometriosis mimicking malignancy. J Cutan Pathol 20:87-91

Paggetti J, Haderk F, Seiffert M, Janji B, Distler U, Ammerlaan W, Kim YJ, Adam J, Lichter P, Solary E, Berchem G, Moussay E (2015) Exosomes released by chronic lymphocytic leukemia cells induce the transition of stromal cells into cancer-associated fibroblasts. Blood 126:1106-1117

Pascucci L, Alessandri G, Dall'Aglio C, Mercati F, Coliolo P, Bazzucchi C, Dante S, Petrini S, Curina G, Ceccarelli P (2014) Membrane vesicles mediate pro-angiogenic activity of equine adipose-derived mesenchymal stromal cells. Vet J 202:361-366

Riches A, Campbell E, Borger E, Powis S (2014) Regulation of exosome release from mammary epithelial and breast cancer cells - a new regulatory pathway. Eur J Cancer 50:1025-1034

Rocha AL, Reis FM, Taylor RN (2013) Angiogenesis and endometriosis. Obstet Gynecol Int 2013:859619

Ryan IP, Schriock ED, Taylor RN (1994) Isolation, characterization, and comparison of human endometrial and endometriosis cells in vitro. $\mathrm{J}$ Clin Endocrinol Metab 78:642-649

Salomon C, Torres MJ, Kobayashi M, Scholz-Romero K, Sobrevia L, Dobierzewska A, Illanes SE, Mitchell MD, Rice GE (2014) A gestational profile of placental exosomes in maternal plasma and their effects on endothelial cell migration. PLoS One 9:e98667

Sampson JA (1927) Metastatic or embolic endometriosis, due to the menstrual dissemination of endometrial tissue into the venous circulation. Am J Pathol 3:143

Scarlett K, Pattabiraman V, Barnett P, Liu D, Anderson LM (2014) Proangiogenic effect of iroquois homeobox transcription factor Irx3 in human microvascular endothelial cells. J Biol Chem 290:6303-6315

Schneider A, Simons M (2013) Exosomes: vesicular carriers for intercellular communication in neurodegenerative disorders. Cell Tissue Res 352:33-47

Schneider CA, Rasband WS, Eliceiri KW (2012) NIH Image to ImageJ: 25 years of image analysis. Nat Methods 9:671-675
Shabbir A, Cox A, Rodriguez-Menocal L, Salgado M, Van Badiavas E (2015) Mesenchymal stem cell exosomes induce proliferation and migration of normal and chronic wound fibroblasts, and enhance angiogenesis in vitro. Stem Cells Dev 24:1635-1647

Shifren JL, Tseng JF, Zaloudek CJ, Ryan IP, Meng YG, Ferrara N, Jaffe RB, Taylor RN (1996) Ovarian steroid regulation of vascular endothelial growth factor in the human endometrium: implications for angiogenesis during the menstrual cycle and in the pathogenesis of endometriosis. J Clin Endocrinol Metab 81:3112-3118

Simpson RJ, Jensen SS, Lim JW (2008) Proteomic profiling of exosomes: current perspectives. Proteomics 8:4083-4099

Swift JL, Sergeev M, Wiseman PW (2010) Fluorescence microscopy investigations of ligand propagation and accessibility under adherent cells. Biointerphases 5:139-148

Tang MK, Wong AS (2015) Exosomes: emerging biomarkers and targets for ovarian cancer. Cancer Lett 367:26-33

Valadi H, Ekstrom K, Bossios A, Sjostrand M, Lee JJ, Lotvall JO (2007) Exosome-mediated transfer of mRNAs and microRNAs is a novel mechanism of genetic exchange between cells. Nat Cell Biol 9:654 659

Verkauf BS (1987) Incidence, symptoms, and signs of endometriosis in fertile and infertile women. J Fla Med Assoc 74:671-675

Wang S, Olson EN (2009) AngiomiRs - key regulators of angiogenesis. Curr Opin Genet Dev 19:205-211

Wang N, Liu B, Liang L, Wu Y, Xie H, Huang J, Guo X, Tan J, Zhan X, Liu Y, Wang L, Ke P (2014) Antiangiogenesis therapy of endometriosis using PAMAM as a gene vector in a noninvasive animal model. Biomed Res Int 2014:546479

Xu Y, Luo F, Liu Y, Shi L, Lu X, Xu W, Liu Q (2015) Exosomal miR-21 derived from arsenite-transformed human bronchial epithelial cells promotes cell proliferation associated with arsenite carcinogenesis. Arch Toxicol 89:1071-1082

Yu J, Berga SL, Zou W, Sun HY, Johnston-MacAnanny E, Yalcinkaya T, Sidell N, Bagchi IC, Bagchi MK, Taylor RN (2014) Gap junction blockade induces apoptosis in human endometrial stromal cells. Mol Reprod Dev 81:666-675 